\title{
Late time evolution of negatively curved FLRW models
}

\author{
Roberto Giambò $^{* 1,2}$, John Miritzis ${ }^{\dagger 3}$ and Annagiulia Pezzola ${ }^{\ddagger 1,4}$ \\ ${ }^{1}$ School of Science and Technology, Mathematics Division, University of \\ Camerino (Italy) \\ ${ }^{2}$ INFN, Sezione di Perugia, 06123-Perugia (Italy) \\ ${ }^{3}$ Department of Marine Sciences, University of the Aegean (Greece) \\ ${ }^{4} \mathrm{PhD}$ School, University of Macerata (Italy)
}

April 24, 2020

\begin{abstract}
We study the late time evolution of negatively curved Friedmann-Lemaitre-Robertson-Walker (FLRW) models with a perfect fluid matter source and a scalar field nonminimally coupled to matter. Since, under mild assumptions on the potential $V$, it is already known - see e.g. [18 - that equilibria corresponding to non-negative local minima for $V$ are asymptotically stable, we classify all cases where one of the energy components eventually dominates. In particular for nondegenerate minima with zero critical value, we rigorously prove that if $\gamma$, the parameter of the equation of state is larger than $2 / 3$, then there is a transfer of energy from the fluid and the scalar field to the energy density of the scalar curvature. Thus, the scalar curvature, if present, has
\end{abstract}

*roberto.giambo@unicam.it

†imyr@aegean.gr

‡annagiulia.pezzola@studenti.unicam.it, a.pezzola1@unimc.it 
a dominant effect on the late evolution of the universe and eventually dominates over both the perfect fluid and the scalar field. The analysis in complemented with the case where $V$ is exponential and therefore the scalar field diverges to infinity.

\section{Introduction}

Scalar fields implement a useful tool used by theorists for the description of the early inflationary phase and of the present accelerating expansion of the Universe, [1, 2, 3]. Scalar fields arise in several conformally equivalent theories of gravity, e.g., in higher order gravity theories (HOG), in string theories [4] and in scalar-tensor theories involving scalar field self-interactions and dynamical couplings to matter [5]. An important example of a scalar field coupled to matter is provided by the general form of scalar-tensor theories of gravity [5, 6, 7], where the action in the Einstein frame takes the form

$$
S=\int d^{4} x \sqrt{-g}\left\{R-\left[(\partial \phi)^{2}+2 V(\phi)\right]+2 \chi^{-2} L_{\mathrm{m}}\left(\widetilde{g}_{\mu \nu}, \Psi\right)\right\}
$$

where

$$
\widetilde{g}_{\mu v}=\chi^{-1} g_{\mu v}
$$

and $\chi=\chi(\phi)$ is the coupling function; matter fields are collectively denoted by $\Psi$. This action contains as special cases HOG theories with $\chi(\phi)=e^{\sqrt{2 / 3} \phi}$ and $\widetilde{g}_{\mu v}=e^{-\sqrt{2 / 3} \phi} g_{\mu v}$. Non minimally coupling occurs also in models of chameleon gravity [8, 9], with $\widetilde{g}_{\mu v}=e^{2 \beta \phi} g_{\mu v}$, where $\beta$ is a coupling constant. The same form of coupling has been proposed in models of the so called coupled quintessence [10] (see also [11, 12, 13] for more general couplings).

Variation of the action (1) with respect to the metric $g$ yields the field equations,

$$
G_{\mu \nu}=T_{\mu \nu}(g, \phi)+T_{\mu \nu}^{\mathrm{m}}(g, \Psi)
$$

where $T_{\mu \nu}^{\mathrm{m}}$ is the matter energy momentum tensor. The Bianchi identities imply that the total energy-momentum tensor is conserved and therefore there is an energy exchange between the scalar field and ordinary matter. In all the above examples, the conservation of 
their sum is provided by the equations (compare to [10]),

$$
\nabla^{\mu} T_{\mu \nu}^{\mathrm{m}}(g, \Psi)=Q T^{\mathrm{m}} \nabla_{\nu} \phi, \quad \nabla^{\mu} T_{\mu \nu}(g, \phi)=-Q T^{\mathrm{m}} \nabla_{\nu} \phi,
$$

where $Q:=d \ln \chi / d \phi$, depends in general on $\phi$ and $T^{\mathrm{m}}$ is the trace of the matter energymomentum tensor, i.e., $T^{\mathrm{m}}=g^{\mu v} T_{\mu v}^{\mathrm{m}}(g, \Psi)$. Variation of $S$ with respect to $\phi$ yields the equation of motion of the scalar field,

$$
\square \phi-\frac{d V}{d \phi}=-Q T^{\mathrm{m}}
$$

In early investigations in scalar-field cosmology a minimal coupling of the scalar field was assumed, (see for example the review articles [3, 7] and references therein). It is true that inclusion of non minimal coupling increases the mathematical difficulty of the analysis; however, it is important to consider non minimal coupling in scalar field cosmology [5]. Many physical theories predict the presence of a scalar field coupled to matter and therefore, the introduction of non minimal coupling is not a matter of taste [14]. Models with exponential potentials have been intensively studied not only because of the variety of alternative theories of gravity which predict exponential potentials, but also due to the fact that this potential has the nice property that $V^{\prime} \propto V$ which allows for the introduction of normalized variables according to the formalism of Wainwright et al [15] - see however, [16, 17, 18, 19]. Another large class of potentials used in scalar-field cosmological models has a local minimum. In view of the unknown nature of the scalar field supposed to cause accelerated expansion, it is important to investigate the general properties shared by all Friedmann-Lemaître-Robertson-Walker (FLRW) models with a scalar field irrespective of the particular form of the potential.

In this paper we study the late time evolution of initially expanding negatively curved FLRW models with a scalar field having an arbitrary bounded from below potential function $V(\phi)$. Although most of the literature deals with the flat case, negatively curved FLRW models cannot be ruled out by current observation in principle, and have been already analysed i.e. in [22] for the massless case (see also final section 4 below). Our results are rigorously proved and do not depend on the specific form of the potential function, but possibly at most on its local form near its minimum. Ordinary matter is described by a 
barotropic fluid with equation of state

$$
p=(\gamma-1) \rho, \quad 0<\gamma<2
$$

The scalar field is nonminimally coupled to matter according to (1), (2) and (4). Under general assumptions on the potential function $V(\phi)$ we study the late time mutual behavior of the energies associated to the scalar and the fluid, and their relation with the "energy" associated to the spatial curvature of the cosmological model. In particular, in case of a nondegenerate minimum of the potential with vanishing critical value, we show that for $\gamma<2 / 3$ the perfect fluid eventually dominates the energy density of the scalar field, i.e., $\Omega_{\rho} \rightarrow 1, \Omega_{\phi}, \Omega_{k} \rightarrow 0$, a property already shared by flat models for $\gamma<1$. However, if $\gamma>2 / 3$, the energy density of the scalar curvature eventually dominates over both the perfect fluid and the scalar field, i.e., $\Omega_{k} \rightarrow 1$ and $\Omega_{\phi}, \Omega_{\rho} \rightarrow 0$ asymptotically. This result shows that the scalar curvature, if present, has a dominant effect on the late evolution of the universe.

The paper is organized as follows. Section 2 briefly recollects the basic ideas of the model studied. The results on the asymptotic behavior of the energy are described in Section 3. In paragraph 3.1 we consider the case when the scalar approaches a local nondegenerate minimum of the potential $V(\phi)$, and find in Theorem 3.1 the spectrum of the qualitative behavior of the energies in terms of the parameter $\gamma$ entering the equation of state of the fluid. To complete the analysis in analogy with [18], we also present in paragraph 3.2 the case of the exponential potential, finding out that the curvature energy may take over the other energies also in this case. The final Section 4 is devoted to conclusions and perspectives.

\section{Model description}

The metric is given by the general, possibly nonflat FLRW metric

$$
\mathrm{d} s^{2}=-\mathrm{d} t^{2}+a(t)^{2}\left(\frac{\mathrm{d} r^{2}}{1-k r^{2}}+r^{2} \mathrm{~d} \Omega^{2}\right)
$$

where $\mathrm{d} \Omega^{2}=\mathrm{d} \vartheta^{2}+\sin ^{2} \vartheta \mathrm{d} \varphi^{2}$. For this metric, assuming that $L_{m}$ is the Lagrangian of a perfect fluid with equation of state (5), the field equations (2) reduce to the Friedmann 
equation,

$$
H^{2}+\frac{k}{a^{2}}=\frac{1}{3}\left(\rho+\frac{1}{2} \dot{\phi}^{2}+V(\phi)\right)
$$

and the Raychaudhuri equation,

$$
\dot{H}=-\frac{1}{2} \dot{\phi}^{2}-\frac{\gamma}{2} \rho+\frac{k}{a^{2}}
$$

while the equation of motion of the scalar field (4), becomes

$$
\ddot{\phi}+V^{\prime}(\phi)+3 H \phi+Q(\phi) \frac{(3 \gamma-4)}{2} \rho=0
$$

where $Q(\phi)$ is the logarithmic derivative of the coupling function $\chi(\phi)$ which is supposed to be strictly positive and differentiable. The Bianchi identities 3 yield the conservation equation,

$$
\dot{\rho}+3 \gamma \rho H=Q(\phi) \frac{4-3 \gamma}{2} \rho \dot{\phi}
$$

Here, $a(t)$ is the scale factor, an overdot denotes differentiation with respect to time $t, H=$ $\dot{a} / a$ and units have been chosen so that $c=1=8 \pi G$. The potential $V(\phi)$ of the scalar field is a $C^{2}$ function and $d V / d \phi$ is denoted by $V^{\prime}(\phi)$.

Setting $y=\dot{\phi}$ and $\alpha(\phi):=\frac{4-3 \gamma}{2} Q(\phi)$, we obtain the system

$$
\begin{aligned}
\dot{\phi} & =y, \\
\dot{y} & =-3 H y-V^{\prime}(\phi)+\alpha \rho, \\
\dot{\rho} & =-3 \gamma \rho H-\alpha \rho y, \\
\dot{H} & =-\frac{1}{2} y^{2}-\frac{\gamma}{2} \rho+\frac{k}{a^{2}},
\end{aligned}
$$

subject to the constraint

$$
3 H^{2}+\frac{3 k}{a^{2}}=\rho+\frac{1}{2} y^{2}+V(\phi) .
$$

We begin with some general properties of the system (10) with the constraint (11). Firstly, the system shares the remarkable property of the Einstein equations that, if equation (11) is satisfied at some initial time, then it is satisfied throughout the evolution. Secondly, following the arguments in [20,21], one can show that an initially expanding flat or negatively 
curved universe remains ever-expanding. Thirdly, the third of (10) implies that the set $\rho=0$ is invariant. Therefore, if initially $\rho$ is positive, it remains positive for ever. Furthermore, for $k=0,-1$ the fourth equation of 10 implies $\dot{H}<0$, thus $H$ is a decreasing function of time $t$ and is bounded from below either by 0 or $\left.\sqrt{V\left(\phi_{*}\right) / 3}\right)$ where $V^{\prime}\left(\phi_{*}\right)=0$. We observe that the function $W(t)$ defined as

$$
W(t)=W(\phi(t), y(t), \rho(t), H(t))=H^{2}-\frac{1}{3}\left(\frac{1}{2} y^{2}+V(\phi)+\rho\right)
$$

satisfies the equation

$$
\dot{W}=-2 H W
$$

therefore, $\operatorname{sgn}(W)$ is invariant under the flow of $(10)$ and since

$$
W(t)=-\frac{k}{a^{2}(t)}
$$

it must be $k=-\operatorname{sgn}(W(0))$.

Using the constraint (11) to eliminate $a$, we observe that the critical points of (10) are given by $\left(\phi=\phi_{*}, y=0, \rho=0, H= \pm \sqrt{V\left(\phi_{*}\right) / 3}\right)$ where $V^{\prime}\left(\phi_{*}\right)=0$.

Following [18], we assume two properties for the scalar potential $V(\phi)$ : (i) the (possibly empty) set $\{\phi: V(\phi)<0\}$ is bounded, and (ii) the set of critical points is finite. Under these assumptions one can show - see e.g. [18, Proposition 1] - that $\left(\phi_{*}, y_{*}=0, \rho_{*}=0, H_{*}=\right.$ $\left.\sqrt{V\left(\phi_{*}\right) / 3}\right)$, where $\phi_{*}$ is a - possibly degenerate - strict local minimum for the potential $V(\phi)$, is an asymptotically stable equilibrium point for expanding cosmologies in the open spatial topologies $k=0$ and $k=-1$. The purpose of this paper is to establish some results on the asymptotic behaviour in the case $k=-1$.

\section{Asymptotic behavior of the energy}

In the following, we are going to study the late time behaviour of solutions of (10), which are initially expanding, i.e., $H(0)>0$. Our aim is to study which is the asymptotically dominating energy in the above model: we have the energy $\rho$ associated to the perfect fluid and 
the energy associated to the scalar field

$$
\epsilon=\frac{1}{2} y^{2}+V(\phi)
$$

so that using equation (12) we have

$$
\Omega_{\rho}+\Omega_{\phi}+\Omega_{k}=1
$$

where

$$
\Omega_{\rho}=\frac{\rho}{3 H^{2}}, \quad \Omega_{\phi}=\frac{\epsilon}{3 H^{2}}, \quad \Omega_{k}=\frac{W}{H^{2}}=\frac{1}{a^{2} H^{2}},
$$

are the normalized energies related to the perfect fluid, the scalar field and the spatial scalar curvature. Notice that the third component may become relevant, unlike the flat case $k=0$ where the leading contribution is either given by the perfect fluid or the scalar. We will consider in subsection 3.1 the case of a nondegenerate minimum of the potential and show the main result of this paper, Theorem 3.1, whereas in subsection 3.2 we will take into account the exponential potential with critical point at infinity.

\subsection{Nondegenerate minimum of the potential}

Let us study the asymptotic behaviour when the scalar field approaches a local minimum of the potential $V(\phi)$. We will focus on the case when $V\left(\phi_{*}\right)=0$ - indeed, when $V\left(\phi_{*}\right)>0$, the energy density of the scalar field approaches a strictly positive value and eventually dominates. The less trivial case is given when the critical value for the potential is zero, and we consider the case where this minimum is nondegenerate. Furthermore, we assume without loss of generality that $\phi_{*}=0$ and therefore, the potential near its minimum takes the form

$$
V(\phi)=\frac{1}{2} \lambda^{2} \phi^{2}+O\left(\phi^{3}\right), \quad \lambda>0 .
$$

From now on, the higher order terms in $V(\phi)$ will be systematically neglected, since it can be shown that the results we are going to state are not affected.

We recall [18, Theorem 2] that, in the flat case $k=0, \Omega_{\rho}$ eventually dominates when $\gamma<1$, whereas $\Omega_{\phi}$ eventually dominates when $\gamma>1$ in a generic way, i.e. except at most for a particular solution of the system. Let us go and see what happens when $k=-1$ and, in 
principle, another form of "energy" deriving from the spatial curvature of the cosmological model enters into play.

Using (6) we can eliminate $k$ from (10) thereby obtaining

$$
\begin{aligned}
\dot{\phi} & =y, \\
\dot{y} & =-3 H y-\lambda^{2} \phi+\alpha \rho, \\
\dot{\rho} & =-3 \gamma \rho H-\alpha \rho y, \\
\dot{H} & =-\frac{1}{3} y^{2}+\left(\frac{1}{3}-\frac{\gamma}{2}\right) \rho+\frac{1}{6} \lambda^{2} \phi^{2}-H^{2} .
\end{aligned}
$$

The phase space of the system is the set

$$
\left\{(\phi, y, \rho, H) \in \mathbb{R}^{4}: H^{2}>\frac{1}{3}\left(\rho+\frac{1}{2} y^{2}+V(\phi)\right)\right\},
$$

due to the constraint (11).

To tackle the problem we consider expansion-normalized variables, a traditionally useful approach for flat cosmologies (see e.g. [17]) that will be exploited here also for the $k=-1$ case under examination. We set

$$
w=\frac{\lambda}{\sqrt{6}} \frac{\phi}{H}, \quad z=\frac{1}{\sqrt{6}} \frac{y}{H}, \quad u=\frac{\sqrt{W}}{H},
$$

in order to write normalized energies (16) as

$$
\Omega_{\rho}=1-\left(u^{2}+w^{2}+z^{2}\right), \quad \Omega_{\phi}=w^{2}+z^{2}, \quad \Omega_{k}=u^{2},
$$


and the system (18) takes the form

$$
\begin{aligned}
& \dot{w}=\lambda z+w H\left(3 z^{2}+u^{2}+\frac{3}{2} \gamma\left(1-w^{2}-z^{2}-u^{2}\right)\right), \\
& \dot{z}=-\lambda w+z H\left(-3+3 z^{2}+u^{2}\right)+3 H\left(1-w^{2}-z^{2}-u^{2}\right)\left(\frac{\gamma}{2} z+\frac{\alpha}{\sqrt{6}}\right), \\
& \dot{u}=-u H\left(1-3 z^{2}-u^{2}-\frac{3}{2} \gamma\left(1-w^{2}-z^{2}-u^{2}\right)\right), \\
& \dot{H}=-H^{2}\left(3 z^{2}+u^{2}+\frac{3}{2} \gamma\left(1-w^{2}-z^{2}-u^{2}\right)\right) .
\end{aligned}
$$

The above system is slightly complicated, with respect to its flat counterpart, by the introduction of one more equation, the third one, that in the flat $k=0$ is trivially satisfied since $W=0$ and then $u=0$. The main complication arising will be that, in the argument below, we will have to employ spherical coordinates instead of polar ones.

We also observe that, in view of the first of (20), and recalling that $\alpha$ is in principle a function of the scalar field $\phi$ only, we can consider $\alpha=\alpha(w, H)$; however one should bear in mind that we are considering solutions such that $\phi \rightarrow \phi^{*}$, and then $\alpha$ will approach a constant as $t \rightarrow+\infty$. This fact will be used in the proofs throughout below.

Finally we notice that, unlike the case that will be treated in next paragraph 3.2 , the presence of the terms $\lambda z$ and $-\lambda w$ in the first two equations of (22) does not allow to consider, as is usually done for similar studies, a normalized time $\tau$, and therefore the first three equations no more decouple from the fourth one, as happens in the exponential case.

As mentioned above, it will be useful to consider the system in spherical coordinates $(R, \theta, \eta)$ defined as

$$
\begin{aligned}
u & =R \cos \eta, \\
w & =R \sin \eta \cos \theta, \\
z & =R \sin \eta \sin \theta,
\end{aligned}
$$


and the system is rewritten in the following form:

$$
\begin{aligned}
\dot{\theta}+\lambda & =3 H \cos \theta\left(-\sin \theta+\frac{\alpha}{\sqrt{6} \sin \eta} \frac{\left(1-R^{2}\right)}{R}\right), \\
\dot{\eta} & =H \cos \eta\left(\sin \eta\left(1-3 \sin ^{2} \theta\right)+\frac{3 \alpha \sin \theta}{\sqrt{6}} \frac{\left(1-R^{2}\right)}{R}\right), \\
\dot{R} & =H\left(1-R^{2}\right)\left(R\left(\sin ^{2} \eta\left(1-3 \sin ^{2} \theta\right)-1+\frac{3 \gamma}{2}\right)+\frac{3 \alpha \sin \eta \sin \theta}{\sqrt{6}}\right), \\
\dot{H} & =H^{2}\left(R^{2}\left(\sin ^{2} \eta\left(1-3 \sin ^{2} \theta\right)-1\right)-\frac{3 \gamma}{2}\left(1-R^{2}\right)\right),
\end{aligned}
$$

compare with [18, eq.(23)]. Notice that, using (21) and (23), the normalized energies now are given by

$$
\Omega_{\rho}=1-R^{2}, \quad \Omega_{\phi}=R^{2} \sin ^{2} \eta, \quad \Omega_{k}=R^{2} \cos ^{2} \eta,
$$

The main result of this paper is the following:

Theorem 3.1. Let $\phi_{*}$ be a nondegenerate minimum of $V(\phi)$ with zero critical value. Consider the solutions of (10) with $k=-1$ approaching the (asymptotically stable) equilibrium point $\left(\phi_{*}, y=0, \rho=0, H=0\right)$. Then if $\gamma<2 / 3$ then the normalized energy of the perfect fluid dominates asymptotically:

$$
\Omega_{\rho} \rightarrow 1, \quad \Omega_{\phi}, \Omega_{k} \rightarrow 0 .
$$

On the other side, when $\gamma>2 / 3$, the energy of the scalar curvature dominates asymptotically:

$$
\Omega_{k} \rightarrow 1, \quad \Omega_{\phi}, \Omega_{\rho} \rightarrow 0
$$

Observe that, in particular, the energy associated to the scalar field never eventually dominates 1 .

To show the above result we consider the compact and positively invariant set $\mathscr{U}=$ $\left\{u^{2}+w^{2}+z^{2} \leq 1, u \geq 0\right\} \times\left\{H \in\left[0, H_{0}\right]\right\}$. By LaSalle's theorem the possible $\omega$-limit points for trajectories living in $\mathscr{U}$ are given by the circles $w^{2}+z^{2}=r_{\infty}^{2}$ (=constant) with $u=u_{\infty}$ constant. We must then observe that a trajectory of the system can admit only one such $\omega$-limit

\footnotetext{
${ }^{1}$ Also observe that the transition case $\gamma=\frac{2}{3}$ is excluded from the current analysis, similarly to the transition case $\gamma=1$ of [18, Theorem 2]
} 
circle, otherwise with a simple contradiction argument one would obtain infinite limit circles of this kind. Therefore, with reference to the variable change given by (23), we find that $\exists \lim _{t \rightarrow+\infty} R(t)=: R_{\infty} \geq 0$ and, if $R_{\infty}>0$, also $\exists \lim _{t \rightarrow+\infty} \eta(t)=\eta_{\infty} \in \mathbb{R}$. Then Theorem 3.1 is a straightforward consequence of the following two claims, that will be shown to hold.

Claim 3.1. If $R_{\infty}=0$ then $\gamma<2 / 3$.

Claim 3.2. If $R_{\infty}>0$ and $\gamma \neq 2 / 3$ then $\gamma>2 / 3$ and $R_{\infty}=1$.

Claim 3.1 holds because, if $R \rightarrow 0$, then from 24d we get that $\dot{H} \approx-\frac{3 \gamma}{2} H^{2}$, and integrating twice we obtain $a \approx t^{\frac{2}{3 \gamma}}$. Since $\dot{a} \approx \frac{2}{3 \gamma} t^{\frac{2}{3 \gamma}-1}$, then it must be

$$
\Omega_{k}=R^{2} \cos ^{2} \eta=u^{2}=\frac{W}{H^{2}}=\frac{1}{\dot{a}^{2}} \approx \frac{9}{4} \gamma^{2} t^{2\left(1-\frac{2}{3 \gamma}\right)},
$$

which recalling $R \rightarrow 0$ is consistent only when $\gamma<2 / 3$.

To prove Claim 3.2 we begin by showing by contradiction that $\sin \eta_{\infty}=0$. Indeed if that is not the case, since $H$ monotonically goes to zero, from 24a we obtain $\theta \approx-\lambda t$ and consequently the following asymptotic estimates as $t \rightarrow+\infty$ are found:

$$
\int_{0}^{t} H \cos \eta \sin \eta\left(1-3 \sin ^{2} \theta\right) \mathrm{d} s=\int_{0}^{t} H \cos \eta \sin \eta\left(\frac{3}{2} \cos 2 \theta-\frac{1}{2}\right) \mathrm{d} s \approx \int_{0}^{t}-\frac{1}{2} H \cos \eta_{\infty} \sin \eta_{\infty} \mathrm{d} s+c_{0}
$$

and

$$
\int_{0}^{t} H \cos \eta \frac{3 \alpha \sin \theta}{\sqrt{6}} \frac{\left(1-R^{2}\right)}{R} \mathrm{~d} s \approx c_{1} \in \mathbb{R},
$$

for some $c_{0}, c_{1} \in \mathbb{R}$. Using the above estimates in 24b we get

$$
\eta(t) \approx-\frac{1}{2} \cos \eta_{\infty} \sin \eta_{\infty} \ln a(t)+c_{2}
$$

for some $c_{2} \in \mathbb{R}$, and therefore it must be $\cos \eta_{\infty}=0$ and so $\sin ^{2} \eta_{\infty}=1$. This implies the following asymptotic estimate in (24d):

$$
\int_{0}^{t} \frac{\dot{H}}{H^{2}} \mathrm{~d} s \approx-v t
$$


where $v=\left(\frac{3}{2} R_{\infty}^{2}+\frac{3}{2} \gamma\left(1-R_{\infty}^{2}\right)\right)$. Integrating once again we find that

$$
u^{2}=\frac{1}{\dot{a}^{2}} \approx t^{2(1-1 / v)}
$$

and recalling that $u^{2}=R^{2} \cos ^{2} \eta \rightarrow 0$ it must be $v<1$, that implies $\gamma<2 / 3$ and $R_{\infty}<\sqrt{2 / 3}$. But similar arguments applied to 24c give

$$
R(t)-R(0) \approx \int_{0}^{t} H\left(1-R_{\infty}^{2}\right) \frac{3}{2} R_{\infty}(\gamma-1) \mathrm{d} s+c_{3}
$$

for some $c_{3} \in \mathbb{R}$, and then either $\gamma=1$ or $R_{\infty}=1$, that are both inconsistent with what we have found above. Then $\sin \eta_{\infty}=0$. Now, recalling that we are excluding $\gamma=2 / 3$, 24c again produces the asymptotic estimate

$$
\frac{\dot{R}}{R\left(1-R^{2}\right)} \approx H\left(\frac{3}{2} \gamma-1\right)
$$

that implies $R / \sqrt{1-R^{2}} \approx a^{\frac{3}{2} \gamma-1}$, which is consistent with $R_{\infty}>0$ only if $\gamma>2 / 3$ and $R_{\infty}=1$, finally proving Claim 3.2 .

Two remarks are in order. Firstly, our results hold also for very weak or even zero coupling, i.e. when $\alpha \rightarrow 0$, see the discussion in the last paragraph of Section 4 . The same conclusion also holds for exponential potentials, see Section 3.2 . Secondly, the asymptotic state when $\Omega_{k} \rightarrow 1$ and $\Omega_{\phi}, \Omega_{\rho} \rightarrow 0$ corresponds to a Milne universe, see also [22]. In that case the asymptotic value of the effective equation of state parameter is $w=-1 / 3$.

The different evolutions described in Theorem 3.1 are illustrated in Figures 1 and 2 In all cases we have considered a coupling function $\chi(\phi)=e^{\sqrt{2 / 3} \phi}$, that gives $\alpha$ constant. The constant value for $\lambda$ in potential (17) has been picked up equal to 2. Moreover initial data have been chosen in such a way that at some initial time, the universe expansion is accelerated in accordance with [23]. Figure 1a shows the evolution of the energies in case $\gamma=1 / 3$ : curvature energy remains small and decreasing to zero, whereas matter energy rapidly dominates over $\Omega_{\phi}$.

The situation is completely different when $\gamma>2 / 3$. In Figure $1 \mathrm{~b}$ is represented the case $\gamma=4 / 3$ : in this case the curvature energy slowly increases and eventually takes over the other energies, with $\Omega_{\rho}$ rapidly vanishing and $\Omega_{\phi}$ decreasing in a more persistent way. 


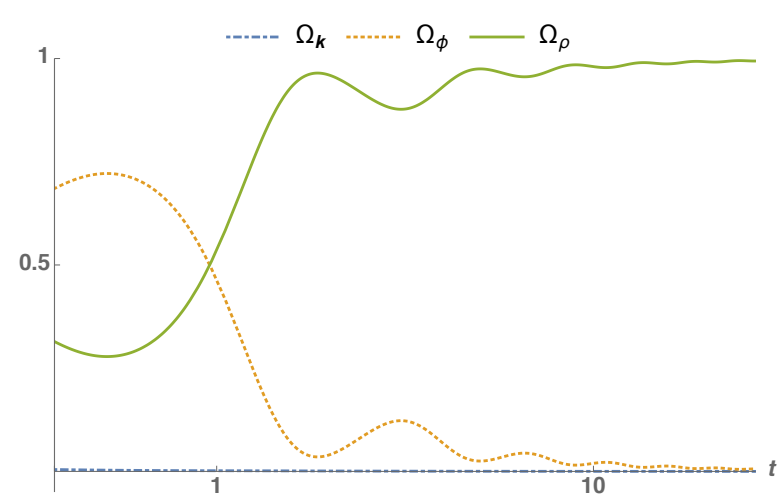

(a) $\gamma=1 / 3$

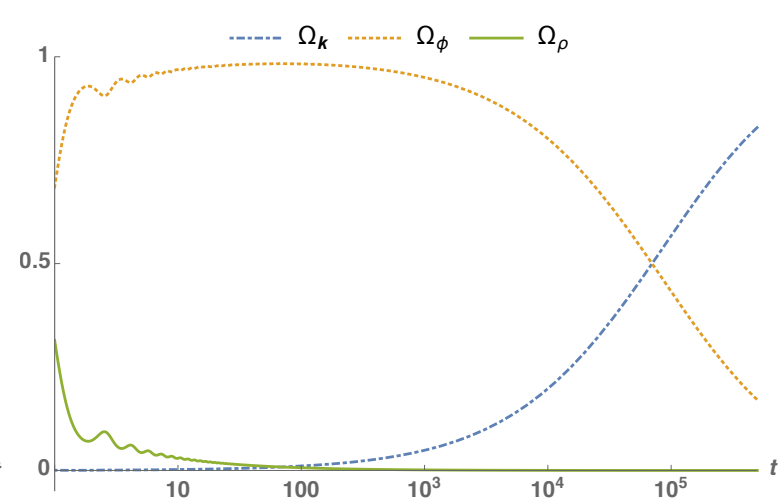

(b) $\gamma=4 / 3$

Figure 1: Normalized energy evolution with coupling function $\chi(\phi)=e^{\sqrt{2 / 3} \phi}$, and potential $V(\phi)=2 \phi^{2}+o\left(\phi^{2}\right)$, corresponding to the two different behaviors found in Theorem 3.1 . Initial data for the normalized energies are $\Omega_{\rho}=0.3145, \Omega_{\phi}=0.685$ and $\Omega_{k}=0.0005$, within the error range expected in [23].

Of course, the way both scalar and matter energies decrease depends on $\gamma$ : see for instance Figure 2 that represents the energy distributions from the same initial data for two cases where pressures are close to zero. As one would expect, $\Omega_{\rho}$ and $\Omega_{\phi}$ go to zero keeping the mutual hierarchy of the flat case $k=0$ which depends on the sign of $\gamma-1$, see [18, Theorem 2].

\subsection{Exponential potential}

To broaden the analysis, let us consider a potential of the form $V(\phi)=V_{0} e^{-\lambda \phi}+\ell$ and assume that there exists a constant $\alpha_{0}$ such that

$$
\alpha_{0}=\lim _{\phi \rightarrow+\infty} \alpha(\phi)=\frac{4-3 \gamma}{2} \lim _{\phi \rightarrow+\infty} Q(\phi) .
$$

As remarked in [18] after the statement of Proposition 1, there is a critical point "at infinity" which is asymptotically stable. If the critical value $\ell$ is strictly positive then the scalar normalized energy approaches 1 , against $\Omega_{\rho}$ and $\Omega_{k}$ which go to zero. The subtle case is then to investigate what happens when $\ell=0$, when $\phi \rightarrow+\infty$, and $y, \rho \rightarrow 0$. Expansion-normalized 


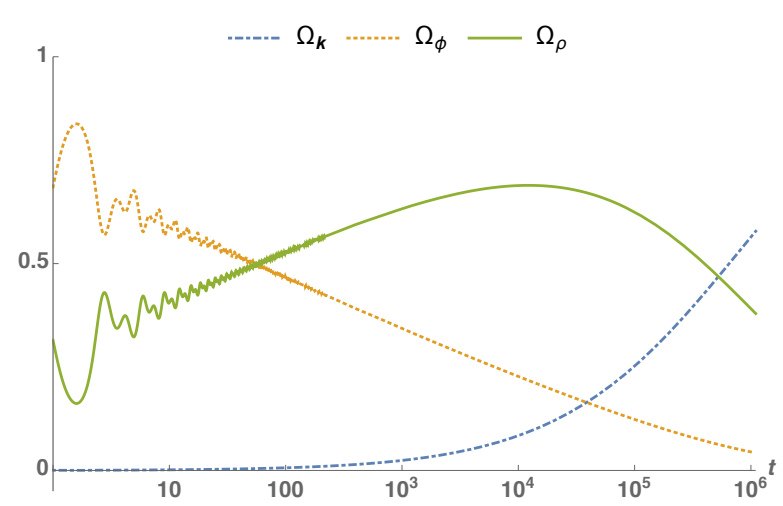

(a) $\gamma=0.9$

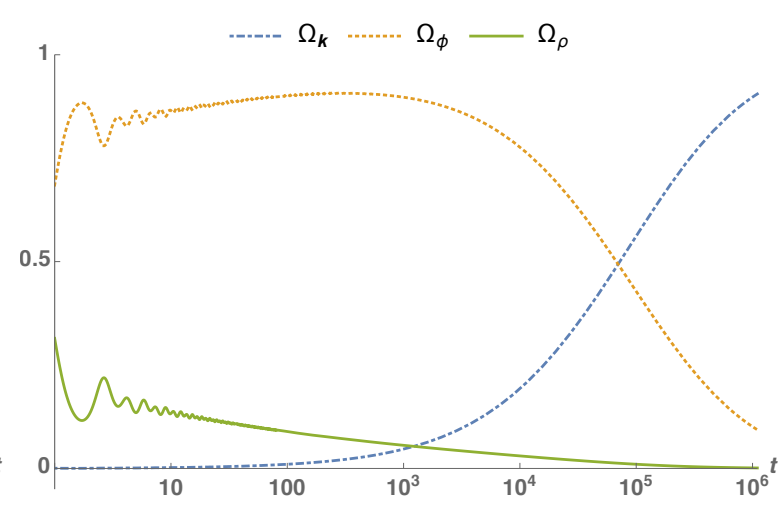

(b) $\gamma=1.1$

Figure 2: Normalized energy evolution for two "near-dust" cases. Here the initial data, coupling function and potential are the same as in Figure 1, and only the value of $\gamma$ changes.

variable change

$$
u=\frac{\sqrt{W}}{H}, \quad w=\sqrt{\frac{V_{0}}{3}} \frac{e^{-\frac{\lambda}{2} \phi}}{H}, \quad z=\frac{y}{\sqrt{6} H},
$$

together with the new time variable defined by $\mathrm{d} \tau=3 \mathrm{Hd} t$ bring system (10) to the form

$$
\begin{aligned}
u^{\prime} & =-\frac{1}{6} u\left(-3 \gamma+(3 \gamma-2) u^{2}+3 \gamma w^{2}+3 \gamma z^{2}-6 z^{2}+2\right) \\
w^{\prime} & =-\frac{1}{6} w\left(-3 \gamma+(3 \gamma-2) u^{2}+3 \gamma w^{2}+3 \gamma z^{2}+\sqrt{6} \lambda z-6 z^{2}\right), \\
z^{\prime} & =z\left(-1+z^{2}+\frac{u^{2}}{3}+\frac{\gamma}{2}\left(1-u^{2}-w^{2}-z^{2}\right)\right)+\frac{1}{\sqrt{6}}\left(\lambda w^{2}+\alpha\left(1-u^{2}-w^{2}-z^{2}\right)\right), \\
H^{\prime} & =-H\left(\frac{1}{3}\left(u^{2}+3 z^{2}\right)+\frac{\gamma}{2}\left(1-u^{2}-w^{2}-z^{2}\right)\right) .
\end{aligned}
$$

Here a prime $\left(^{\prime}\right)$ denotes differentiation with respect to the new time $\tau$. In this case the evolution equation for $H$ decouples from the rest of the evolution equations and so we have a system of three equations in the unknowns $u, w, z$. The definition (27) implies that the variables $u, w, z$ are all positive, included $z$; indeed we know that $\phi \rightarrow+\infty$, therefore situations where $y$ is eventually negative are irrelevant for our study.

A careful inspection of the asymptotically stable equilibria $\left(u_{\infty}, w_{\infty}, z_{\infty}\right)$ of $(28$ ) gives the following five mutual exclusive situations, depending on the values of the three parameters 
$\lambda>0, \gamma \in(0,2), \alpha_{0} \in \mathbb{R}:$

$$
\begin{aligned}
& \mathscr{P}_{1}=\left(0, \sqrt{1-\frac{\lambda^{2}}{6}}, \frac{\lambda}{\sqrt{6}}\right), \mathscr{P}_{2}=\left(\frac{\sqrt{\lambda^{2}-2}}{\lambda}, \frac{2}{\sqrt{3} \lambda}, \frac{\sqrt{\frac{2}{3}}}{\lambda}\right), \mathscr{P}_{3}=\left(0,0, \frac{\sqrt{\frac{2}{3}} \alpha_{0}}{2-\gamma}\right), \\
& \mathscr{P}_{4}=\left(\frac{\sqrt{\alpha_{0}^{2}-\frac{3 \gamma^{2}}{2}+4 \gamma-2}}{\alpha_{0}}, 0, \frac{2-3 \gamma}{\sqrt{6} \alpha_{0}}\right), \mathscr{P}_{5}=\left(0, \frac{\sqrt{\alpha_{0}^{2}-\alpha_{0} \lambda-\frac{3}{2}(\gamma-2) \gamma}}{\lambda-\alpha_{0}}, \frac{\sqrt{\frac{3}{2}} \gamma}{\lambda-\alpha_{0}}\right) \text {. }
\end{aligned}
$$

Recalling (21) it is easy to see that in cases $\mathscr{P}_{3}$ and $\mathscr{P}_{5}$ either the scalar energy or the fluid energy eventually dominate, in case $\mathscr{P}_{1}$ the scalar energy eventually totally dominates, whereas in cases $\mathscr{P}_{2}$ and $\mathscr{P}_{4}$ there might be situations where the curvature energy $\Omega_{k}$ eventually dominates.

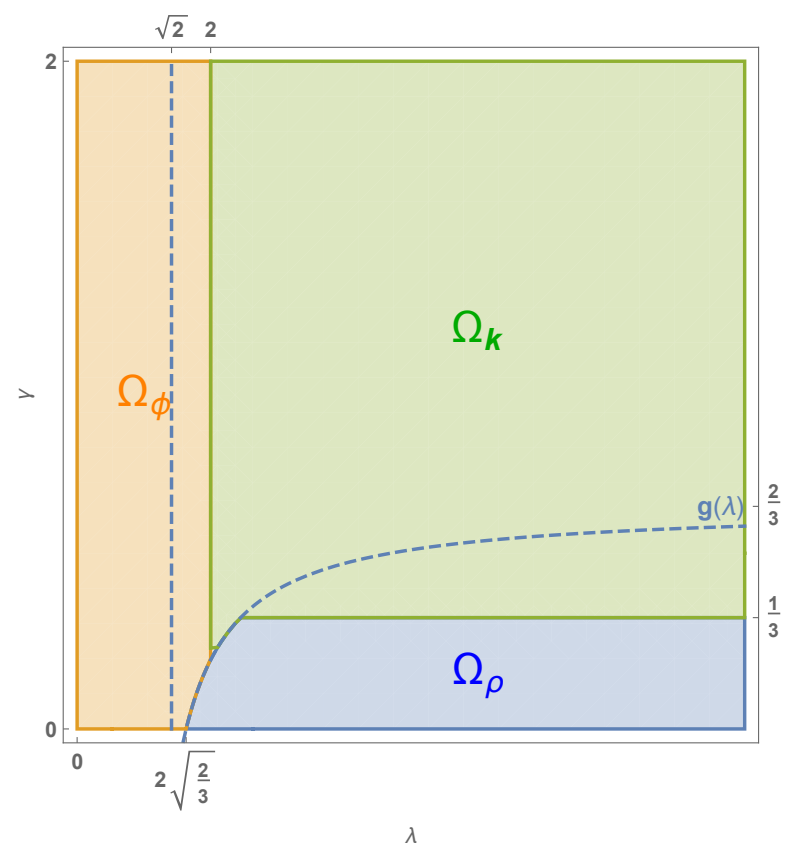

Figure 3: Asymptotically dominating energy when $V=V_{0} e^{-\lambda \phi}$ and the coupling function is given by $\chi(\phi)=e^{\sqrt{2 / 3} \phi}$, in terms of the two free parameters $\lambda, \gamma$. The dashed lines separate the regions where the system (28) approaches the three possibile cases $\mathscr{P}_{1}, \mathscr{P}_{2}$ and $\mathscr{P}_{4}$ $\left(H \rightarrow 0^{+}\right.$always).

Moreover, the scale factor behaves for $t \rightarrow+\infty$ as

$$
a(t) \approx t^{p}, \quad p=\left(u_{\infty}^{2}+3 z_{\infty}^{2}+\frac{3}{2} \gamma\left(1-u_{\infty}^{2}-w_{\infty}^{2}-z_{\infty}^{2}\right)\right)^{-1}
$$


As an example, let us give the detailed results when $\chi(\phi)=e^{\sqrt{2 / 3} \phi}$, so that $\alpha=\alpha_{0}=$ $(4-3 \gamma) \sqrt{6}$. In this case only the three cases $\mathscr{P}_{1}, \mathscr{P}_{2}$ and $\mathscr{P}_{4}$ may take place, respectively:

- $\mathscr{P}_{1}: 0<\lambda<\sqrt{2}$, and $a(t) \approx t^{2 / \lambda^{2}}$;

- $\mathscr{P}_{2}: \lambda>\sqrt{2}$ and $\max \{0, g(\lambda)\}<\gamma<2$, where $g(\lambda)=\frac{6 \lambda-4 \sqrt{6}}{9 \lambda-3 \sqrt{6}}$, and $a(t) \approx t$;

- $\mathscr{P}_{4}: \lambda>2 \sqrt{\frac{2}{3}}, 0<\gamma<g(\lambda)$, and $a(t) \approx t$.

Using the fourth equation in (28) we find

$$
\ddot{a}=a\left(H^{2}+3 H H^{\prime}\right)=1-u^{2}-3 z^{2}+\frac{3}{2} \gamma\left(u^{2}+w^{2}+z^{2}-1\right),
$$

and therefore examples with positive acceleration at initial data can be easily provided.

Figure 3 represent the strip $(\lambda, \gamma) \in(0,+\infty) \times(0,2)$ : the dashed lines separate the three regions described above. Using (21) we conclude that there is a wide region of the two parameters where the curvature energy dominates over the other two energies.

\section{Further discussion}

It is well known that astronomical measurements constrain the spatial curvature to be very close to zero, although they do not constrain its sign [24]. For example, results of the Planck mission released in 2015 show the cosmological curvature parameter, $\Omega_{k}$, to be $0.000 \pm 0.005$, consistent with a flat universe. More recent constraints on $\Omega_{k}$, yield the values $0.001 \pm 0.002$, [23]. Similar bounds for $\Omega_{k}$ were found in an analysis of 42 measurements of the Hubble parameter and baryon acoustic oscillation data in [25], although the authors stress that "more and better data are needed before we can make definitive statements about the spatial curvature of the universe". Larger bounds $0.09 \pm 0.19$ for $\Omega_{k}$ were found in a model-independent method to test the curvature of the universe [26]. Although a flat universe is usually assumed when one studies the nature of dark energy [27], for some models of dark energy, an open universe is more favoured than a flat universe [28]. Thus a flat universe may not be a good assumption for constraining some particular models of dark energy. 
In this investigation we analysed the late time evolution of negatively curved expanding FLRW models having a scalar field coupled to matter. For exponential potentials $V(\phi)=$ $V_{0} e^{-\lambda \phi}$, the energy density of the scalar curvature eventually dominates over both the perfect fluid and the scalar field for a wide range of the parameters $\lambda$ and $\gamma$. For non-negative local minima of $V$, the corresponding equilibria share the same properties as in the flat case [18] namely, they are asymptotically stable and in case the minimum is positive, say $V\left(\phi_{*}\right)>0$, the energy density of the scalar field eventually rules over the energy density of the fluid and the asymptotic state has an effective cosmological constant $V\left(\phi_{*}\right)$. In that case, the equilibrium solution represents an accelerating future attractor. In case the minimum is zero and nondegenerate, then $\Omega_{\rho}$ eventually dominates if $\gamma<2 / 3$. However, if $\gamma>2 / 3$, the energy density of the scalar curvature eventually dominates over both the perfect fluid and the scalar field.

One can understand this result by the following heuristic reasoning. Integrating the energy density equation (9), we obtain $\rho=c e^{-\alpha \phi} a^{-3 \gamma}$. Since $\phi \rightarrow \phi_{*}$ as $t \rightarrow \infty$, absorbing $e^{-\alpha \phi_{*}}$ into the constant of integration, we can write $\rho \simeq c a^{-3 \gamma}$ as $t \rightarrow \infty$. If one could use the Kryloff-Bogoliuboff (KB) approximation [29] it could be shown that near the equilibrium the energy density of the scalar field decreases as $\epsilon \simeq a^{-3}$. Since $H^{2} \Omega_{k}$ scales as $a^{-2}$, it is reasonable to conclude that for $\gamma>2 / 3$ it dominates over both $\epsilon$ and $\rho$ at late times. As remarked in [30], the main obstruction in applying KB approximation lies in the impossibility to have an a-priori estimate on $\rho / \epsilon=\Omega_{\rho} / \Omega_{\phi}$, therefore the need of alternative strategies to attack the problem.

The above results were rigorously proved assuming only that critical points are finite and at those points $V(\phi)$ is non-negative. No further assumption on $V$ enter in the study of the late time behavior around a critical point $\phi_{*} \in \mathbb{R}$, because for that situation only the behavior of the potential near $\phi_{*}$ is important and no growth at infinity assumptions on $V$ are actually needed.

A similar behavior as in Theorem 3.1 of the curvature over two interacting fluids was observed in [22] for $k=-1$ models. Recalling that in the massless case the scalar field reduces to a stiff fluid, in view of our result we can say that the $\gamma=2 / 3$ threshold found in [22] is stable with respect to the interaction with a potential having a local minimum with vanishing critical value. The curvature dominated asymptotic state was also found in [31 
where FLRW models with an exponential potential and a barotropic fluid without coupling were studied. Nonflat cosmologies have been analytically studied also in [32], where dark energy is modeled by a cosmological constant and the problem is recast into a competitive species dynamical framework.

An important question that should be further investigated is the case of closed cosmologies [33]. Based on the experimentation with the potential $V(\phi)=V_{0}\left(1-e^{-\sqrt{2 / 3} \phi}\right)^{2}$ which arises in the conformal frame of quadratic gravity [34], we believe that a closed model cannot avoid recollapse, unless the minimum of the potential is strictly positive (see [35] for non interacting fluid and scalar field). In that case, the asymptotic state must be de Sitter space.

An other important issue is the strength of the coupling function $Q(\phi)$. For viable dark energy models, it is necessary that the energy density of the scalar field remains insignificant during most of the history of the universe and emerges only at late times to account for the current acceleration of the universe. However, in models with double exponential potentials it was observed that only a very weak coupling of the scalar field to ordinary matter can lead to acceptable cosmological histories of the universe [36]. This fact reinforces the general conclusions in [37,38, 39], that HOG dark energy models with $f(R)=R-\mu^{2(n+1)} / R^{n}$, where $\mu>0, n>1$, are not cosmologically viable. This result is attributed to the fact that in these theories, matter is strongly coupled to gravity (recall that $Q=\sqrt{2 / 3}$ in HOG theories). Nevertheless, in [40, 41] specific examples of $f(R) \sim R^{n}$ gravity models were built, including matter and accelerated phases which are cosmological viable, at the expense of having noninteger values for $n$. We do not enter into the old discussion about the equivalence issue of the Einstein and the Jordan frame (see for example [42]; see also [43] with specific examples and the extended review articles [44, 45, 46, 47, 48] with references therein). As mentioned after the proof of Theorem 3.1, our results hold for every value of the coupling function, even for $\alpha \rightarrow 0$. Had we allowed a dynamical role to $Q$, it would be very interesting to see if the dynamics leads to a very tiny value of $Q$ at late times. Such a result could lead to a generalization of the attractor mechanism of scalar-tensor theories towards general relativity, found by Damour and Nordtvedt in the case of a massless scalar field [49, 50]. 


\section{Acknowledgement}

The authors wish to thank Salvatore Capozziello and Orlando Luongo for valuable discussions and suggestions. We also thank an anonymous referee for his suggestions which helped us to clarify some points.

\section{References}

[1] A.A. Coley, Dynamical Systems and Cosmology, (Kluwer Academic Publishers, Dordrecht, Boston, London, 2003).

[2] S. Weinberg, Cosmology, (OUP, Oxford, 2008).

[3] S. Bahamonde, C.G. Böhmer, S. Carloni, E.J. Copeland, W. Fang, N. Tamanini, Phys. Rep. 775-777, 1-122, (2018).

[4] M. Gasperini, Elements of String Cosmology, (CUP, Cambridge, 2007).

[5] Y. Fuji, K. Maeda, The Scalar-Tensor Theory of Gravitation, (CUP, Cambridge, 2003); V. Faraoni, Cosmology in Scalar-Tensor Gravity, (Kluwer Academic Publishers, Dordrecht, 2004).

[6] R. Bean, D. Bernat, L. Pogosian, A. Silvestri, M. Trodden, Phys. Rev. D 75 (6), 064020, (2007).

[7] G. Leon, P. Silveira, C.R. Fadragas, arXiv:1009.0689 [gr-qc] (2010).

[8] J. Khoury, A. Weltman, Phys. Rev. D 69, 044026, (2004).

[9] T.P. Waterhouse, arXiv:astro-ph/0611816 (2006).

[10] L. Amendola, Phys. Rev. D 62, 043511, (2000).

[11] A. Pourtsidou, C. Skordis, E.J. Copeland, Phys. Rev. D 88, 083505, (2013); E.J. Copeland, A.R. Liddle, D. Wands, Phys. Rev. D 57, 4686, (1988).

[12] O. Luongo, M. Muccino, Phys Rev D 98, 103520, (2018). 
[13] A.P. Billyard, A.A. Coley, Phys. Rev. D 61, 083503, (2000).

[14] V. Faraoni, Phys. Rev. D 62, 023504, (2000).

[15] J. Wainwright, G.F.R. Ellis, Dynamical Systems in Cosmology, (CUP, Cambridge, 1997).

[16] V.A. Belinsky, I.M. Khalatnikov, L.P. Grishchuk, Y.B. Zeldovich, Phys. Lett. B 155, 232, (1985).

[17] Wei Fang, Ying Li, Kai Zhang, Hui-Qing Lu, Class. Quantum Grav. 26, 155005, (2009).

[18] R. Giambò, J. Miritzis, Class. Quantum Grav. 27, 095003, (2010).

[19] N. Dimakis, A. Karagiorgos, A. Zampeli, A. Paliathanasis, T. Christodoulakis, P.A. Terzis, Phys. Rev. D 93, 123518, (2016).

[20] S. Foster, Class. Quant. Grav. 15, 3485, (1998).

[21] J. Miritzis, Class. Quantum Grav. 20, 2981, (2003).

[22] A. Nunes, J.P. Mimoso, T.C. Charters, Phys. Rev. D 63, 083506, (2001).

[23] N. Aghanim et al, Planck Collaboration, (arXiv:1807.06209 [astro-ph.CO]) (2018).

[24] M. Kowalski et al, The Supernova Cosmology Project, ApJ 686, 749-778, (2008).

[25] J. Ryan, S. Doshi, B. Ratra, Mon. Not. Roy. Astron. Soc. 480 no.1, 759-767, (2018).

[26] H. Yu, F.Y. Wang, ApJ 828, 85, (2016).

[27] G. Ryskin, Astropart. Phys. 62, 258-268, (2015).

[28] K. Ichikawa, M. Kawasaki, T. Sekiguchi, T. Takahashi, J. Cosmol. Astropart. Phys. 12, 005, (2006).

[29] N. Kryloff, N. Bogoliuboff, Introduction to Nonlinear Mechanics, (PUP, Princeton, 1943).

[30] J. Miritzis, R. Giambò, AIP Conf. Proc. 1241, 1061, (2010). 
[31] R.J. van den Hoogen, A.A. Coley, D. Wand, Class. Quantum Grav. 16,1843, (1999).

[32] J. Perez, A. Füzfa, T. Carletti, L. Mélot, L. Guedezounme, Gen. Rel. Grav. 46, 1753, (2014).

[33] C.G. Park, B. Ratra, ApJ 868, 83, (2018).

[34] J. Miritzis, J. Math. Phys. 46, 082502, (2005).

[35] A.A. Coley, M. Goliath, Phys. Rev. D 62, 043526, (2000).

[36] K. Tzanni, J. Miritzis, Phys. Rev. D 89, 103540, (2014).

[37] L. Amendola, D. Polarski, S. Tsujikawa, Phys. Rev. Lett. 98, 131302, (2007).

[38] L. Amendola, R. Gannouji, D. Polarski, S. Tsujikawa, Phys. Rev. D 75, 083504, (2007).

[39] L. Amendola, D. Polarski, S. Tsujikawa, Int. J. Mod. Phys. D 16, 1555, (2007).

[40] S. Capozziello, P. Martin-Moruno, C. Rubano, Phys. Lett. B 664, 12-15, (2008).

[41] S. Capozziello, S. Nojiri, S.D. Odintsov, A. Troisi, Phys. Lett. B 639, 135-143, (2006).

[42] M. Ferraris, M. Francaviglia, G. Magnano, Class. Quantum Grav. 7, 261, (1990); S. Cotsakis, Phys. Rev. D 47, 1437, (1993); Erratum Phys. Rev. D 49, 1145, (1994); S. Capozziello, R. de Ritis, A.A. Marino, Class. Quantum Grav. 14, 3243, (1997); C.H. Brans, Class. Quantum Grav. 5 L, 197, (1998); G. Magnano, L.M. Sokołowski, Phys. Rev. D 50, 5039, (1994); V. Faraoni, E. Gunzig, P. Nardone, Fund. Cosmic Phys. 20, 121, (1999); V. Faraoni, S. Nadeau, Phys. Rev. D 75, 023501, (2007); S. Capozziello, M. Francaviglia, Gen. Relativ. Gravit. 40, 357-420, (2008); G. Leon, Class. Quantum Grav. 26, 035008, (2009).

[43] S. Capozziello, P. Martin-Moruno, C. Rubano, Phys. Lett. B 689, 117-121, (2010).

[44] T. Sotiriou, V. Faraoni, Rev. Mod. Phys. 82, 451-497, (2010).

[45] A. De Felice, S. Tsujikawa, Living Rev. Relativity 13, 3-161, (2010).

[46] S. Nojiri, S.D. Odintsov, Phys. Rep. 505, 59-144, (2011). 
[47] S. Capozziello, M. De Laurentis, Phys. Rep. 509, 167-321, (2011).

[48] S. Nojiri, S.D. Odintsov, V.K. Oikonomou, Phys. Rep. 692, 1-104, (2017).

[49] T. Damour, K. Nordtvedt, Phys. Rev. Lett. 70, 2217, (1993); T. Damour, K. Nordtvedt, Phys. Rev. D 48, 3436, (1993).

[50] I.P. Mimoso, A. Nunes, Astrophys. Space Sci. 283 no. 4, 661, (2003). 